\title{
Innovative Research on the Combination of Traditional Bamboo Weaving Skills and Modern Design in Ruichang
}

\author{
Li Huang \\ Jiangxi Vocational Technical College of Industry \& Trade, Jiangxi, Nanchang330038 \\ 739960103@qq.com
}

\begin{abstract}
As one of the four famous ancient countries in the world, China has a lot of material and intangible cultural heritage. Among them, Ruichang bamboo weaving is a national intangible cultural heritage, which has been inherited for more than 3000 years and belongs to traditional handicraft production. This paper mainly studies the innovation and thinking of the combination of Ruichang bamboo weaving traditional skills and modern design. For traditional handicraft industry, even if it becomes a national intangible cultural heritage, it also needs to pay attention to its own inheritance and development. Therefore, it is necessary to carry out better innovation and inheritance through the combination with modern design.
\end{abstract}

Keywords: Ruichang bamboo weaving, Traditional skills, Modern design

\section{瑞昌竹编传统技艺与现代设计结合的创新性研究}

\section{黄丽}

江西工业贸易职业技术学院 江西 南昌 330038
739960103@qq.com

摘要:

我国作为世界闻名的四大古国之一, 具有很多物质与非物质文化遗产, 其中瑞昌竹编是国家级非物质文化遗 产, 传承至今已经有 3000 多余年, 属于传统的手工业生产。本文主要在研究瑞昌竹编传统技艺与现代设计结 合的创新性与思考。对于传统手工业来说, 即使成为国家级非物质文化遗产, 也需要去注重自身的传承与发 展。因此就需要去通过与现代设计结合来去进行更好的创新与传承，推陈出新。

关键词：瑞昌竹编；传统技艺；现代设计

\section{1. 瑞昌竹编的介绍}

\section{1. 瑞昌竹编的概况}

从瑞昌的地理位置上来说, 所处的地理位置在 长江中下游南岸, 这里也被称为长江的黄金水道。 在江西省管辖区之内有九江市代管, 本身的气候温 和, 其范围内有着大量的植物资源, 其中以竹林资 源所占比例最多。根据数据表明, 竹林面积可以占 整个九江地区的八成左右, 因此丰富的自然资源,
导致瑞昌的竹编即广为流传，最早可以追溯到上周 时期。

瑞昌竹编最早进行竹子加工并之后进入市场 的，在 3000 多年的历史发展情况下，其中以瑞昌竹 编三宝最为出名，其中就包括丝箩、斗笠、米篮。 在 80 年代以前, 在当地颇有盛名。后来由于经济发 展和社会生产力的提高, 导致出现很多新材料的日 用品, 逐渐替代了竹编生产的日用品, 相比于竹编 生产的日用品更加耐用方便而且不易腐坏。

所以瑞昌竹编的产品类型重心也逐渐开始由日 用品转向艺术品发展, 所以发展到现在传统技艺的 
手工业竹编商品在现代市场上仍旧面临着相应的挑 战。

\section{2. 瑞昌竹编的特点}

瑞昌竹编在制作过程中一些细节上的工艺技 巧, 显示着瑞昌竹编匠人的巧妙思维, 所以即使从 表面上看与别的地方有着一定的相似性, 但是仔细 观之却有大量的不同。比如在编制斗笠的过程中, 瑞昌竹编就需要在斗的中间放一层叶子称为斗笠 叶, 这种叶子具有叶面宽大, 韧性好以及耐用而不 透水的特征。正是因为这种特殊材质的原因, 导致 瑞昌竹编的斗笠与别的竹编斗笠有着一定的不同。

但随着社会的发展, 市场的需求不断上升, 瑞 昌竹编从最早的植物为主到现在, 以动物为主开始 围绕动物来进行开发制作。以动物为主题的开发制 作, 在其他江西竹编工艺厂产品中也有着相似的存 在, 但是只有瑞昌竹编的动物主题产品不仅仅拥有 一定的实用性, 还能够在保证实用性基础上加强观 赏性和装饰性, 所以说瑞昌竹编的特点也可以算是 兼顾实用性的同时也把艺术性做好的代表。

\section{3. 瑞昌竹编的发展现状}

伴随着国家的进步, 经济发展逐渐迅速起来, 导致无论是日用品还是工艺品, 在材料的制作方面 都有了翻天覆地的变化。相比于传统的手工业制 作。新型的手工业制作不仅仅有着更方便使用的材 料, 而且还有着能够进行批量生产的机械, 大大降 低了人工制作的成本, 满足了人们的日常需求。所 以瑞昌竹编在制作过程中, 就已经面临着现代工业 化生产批量制造所带来的挑战。

不仅如此, 这也是目前很多传统手工业制作普 遍所面临的挑战有问题。因此对于传统手工业发展 来说, 就需要去考虑如何在目前这样的市场环境下 进行生存和发展。解决这个问题不仅仅只是需要去 把非物质文化遗产的精神进行认知, 还需要对自身 传统手工业的产业进行转化, 从而顺从经济市场发 展。

\section{2. 瑞昌竹编传统技艺可 “再生” 的要素}

\section{1. 瑞昌竹编材料方面}

瑞昌竹编的编织材料, 主要以当地的毛竹为 主。当地的毛竹具有韧性好耐用的特点, 并且一根 竹条一般可以切为八层䉝片, 别的地方的竹子一根 竹条一般只能开四层策片, 所以本地毛竹就有着先 天的优势。而且瑞昌本地的毛竹所得到的主材, 无 论是逐条还是图片都可以在制作过程中保障整个编 制过程中不会呈现断裂的情况。
而且从色泽上来看, 当地模组所编织出来的头 层青策光往往表现为顺滑耐磨, 青翠欲滴, 整体上 的竹子质量都是属于上品以上, 因此瑞昌竹编所采 用的毛竹品质，不仅仅对现代产品中的材料有着更 高的要求, 而且还可能影响未来现代人对颜色受众 方面有着更大的扩展方向, 这就使得瑞昌竹编具有 很大的应用性。

\section{2 . 瑞昌竹编编织技术方面}

从瑞昌竹编的编织技巧方面来看, 其主要的编 织手法主要有平行编、方孔编、六角孔编等等。其 中平行边主要应用于绝大多数的模具编制上, 平行 边的主要技巧在于需要敷在模具的表面上进行编 织, 这种编织手法可以更好的把握整个编织物品的 形状, 所采用的材料也是比较细的䉝条。而且在编 织过程中还需要注意模具形状的走向模具是什么形 状, 最终编出来的产品也是什么形状。所以根据日 常竹编的模具编织法以及简单的编织手法的形态摸 索, 可以了解到单一功能的产品和高超的编织技艺 结合能够更好的挖掘出更有实用和观赏价值完美结 合的产品。

\section{3. 瑞昌竹编造型方面}

在生产过程中瑞昌竹编主要分为生活日用品以 及工艺品两大类造型来进行后续的生产。瑞昌竹编 的生活日用品, 主要产品有储物小管子、籍箕、晒 筐、食盘（盒）、果盘（盒）等，同时在保证使用系 统同时也可以更好的去提高产品功能性的美观度 啊, 就可以在此基础上去增加更多复杂的周边文 件, 这样不仅仅有更好的观赏价值, 还可以加强生 活日用品的质量与质感, 同时这也是区别于机械工 业化批量生产的一个不同点。

工艺品主要是以动物题材为主, 因此这方面的 造型也都是以动物的造型体现出来。虽然是动物的 形态, 但也会根据功能的不同有所区别: 可分为单 纯的工艺欣赏和附加实用功能的工艺品。

从大多数手工业产品的再生角度来进行观察, 不然发现瑞昌竹编的产品中主要还是以功能性产品 为主。这样的功能性产品主要是去添加更多的产品 技术和艺术含量。倘若是以工艺产品为主, 就需要 去考虑产品当中能够附加的更多价值文化价值或者 是地域价值, 都有可能成为更好的发展方向。

\section{3. 现代竹产品的运用情况分析}

\section{1. 现代竹产品的类型}

随着当前我国竹编市场的不断发展, 竹编类的 产品造型也发生了很大的改变, 而人造产品虽然本 身就有着很多的优点, 但由于其并不是天然材料制 成的, 而传统手工业的主要材料多选用纯天然材 
料，这也就成为了传统手工业与机械制造业相比所 具有的优势。

因此, 现代竹产品就可以充分利用这个优势, 同时根据不同材质的特性以及不同消费者的喜好, 制作出符合现代社会需求的产品, 例如可以制作一 些体积较大的如茶叶管、茶席、茶垫、竹编果篮、 收纳盒等, 还可以制作一些小型的如杯垫、茶具、 茶托、点心零食碟以及小饰物等各种竹编类产品。

\section{2. 现代竹产品的特点}

根据现代竹产品涉及到的不同类型, 可以看出 竹类产品的特点主要可以分为三大类:

一是竹类产品的造型更符合现代社会消费者对 产品不断变化的功能需求, 消费者不再只是注重以 往对产品功能的使用, 现在更加关注的是新产品能 不能符合自己对慢节奏生活的需求, 而这就需要在 进行产品设计时多从生活细节以及生活必需品等方 面来寻找灵感;

二是竹类产品的外形也更符合消费者对时尚、 简洁这类形态的偏爱, 消费者也不再只是以往单纯 根据产品的不同功能来进行相对应产品样式的选 择; 三是竹类产品的色泽可以更多的体现出 “复 古”

色, 而这种较为厚重的颜色可以带给消费者更 多的年代感以及温暖感, 在现代社会消费者不再只 是追求与 “求新” 相关的产品理念, 而对于这种 “做旧”技艺已经被越来越多的消费者所喜爱。

\section{3. 现代竹产品的问题}

在现代社会竹类产品大多还属于 “小众产品”, 原因主要可以分为以下三点:

一是因为消费者对使用新型材料的产品目前还 很依赖, 同时由于价格上的优势, 消费者更多的看 到了这类产品的便利, 但忽视了这类人造材料长期 使用有可能会带来的危害;

二是因为竹类产品现有的市场类型大多是与休 闲娱乐相关的生活类产品, 而这类产品的消费群体 本身就比较少, 因此在竹类产品一开始的功能定位 上就缺乏更加广阔的拓展空间;

三是因为从竹类产品更长远的发展来看, 现有 的竹类产品缺乏相应的设计内涵, 关于产品的艺术 和技术都并没有得到更好的结合以及展示, 使得现 有的竹类产品既不满足实用性, 也不符合高雅性。

\section{4 瑞昌竹编与现代设计结合的可行性分析}

\section{1. 材料特性的现代体现}

从瑞昌竹编的现有条件上来看, 其实可以更好 的去利用多方面的拓展, 来增强它的现代体现。比 如在材料选用方面可以去培养更好的竹子材料来增 加材料的㓞性，同时也可以去对材料的颜色进行可 塑性增强。只有这样才能够让材料去影响整个产品 的设计因素, 从而客观整个产品造型的延展性, 打 通更好的产品功能与造型, 创新性的去发展瑞昌竹 编的联动性。

\section{2. 瑞昌竹编产品的功能拓展}

从瑞昌竹编的传统产品功能上来看, 主要可以 分为以下两种, 一是单纯实用功能的编织物, 二是 通过造型来进行动物模仿的使用功能工艺品。两个 方面都可以根据当前市场的需求来进行功能上的拓 展。例如可以通过当前瑞昌主病的发展情况，将以 前单一的使用功能的基础上去附加更多的观赏价 值, 同时也可以根据消费者日常生活的行为特征结 合人们的生活需求，把功能更加的余墨进行结合， 在满足人民需求的同时，做好美观上的设计。

新的产品也可以去借助现代工具机器去提高整 个昨天产品的制作效率，当然是在保证整个质量的 基础上进行。

瑞昌竹编动物造型的工艺品可以说是十分重要 的发展主题方向, 所以必须延续下去, 在技术上去 附加更多的发展途径, 打好自己的品牌效应, 走更 好的质量道路, 明确自身在市场上的定位, 更好的 去促进卫星主场的发展。

\section{3. 设计形式美法则在竹编造型中的运用}

瑞昌竹编与现代设计结合的可行性中主要有三 点。第一个是材料特性的现代体现; 瑞昌竹编产品 的功能拓展以及设计形式美法则在竹编造型中的运 用。这三点使得瑞昌竹编与现代设计结合提供了可 能性。

瑞昌竹编作为传统手工业的代表之一, 能够一 直从历史发展到现在，不仅仅会受到现代技术的影 响, 同样也离不开现代设计的相对渗入。好的现代 技术和现代设计思维, 能够引导日常周边往好的方 向发展。例如竹编的造型往往可以根据目前现代艺 术设计中很多形式美法去进行应用，将当代的工业 理性塑造美的方法, 用于竹编造型, 将竹编的古朴 气息与现代的工业继续进行碰撞, 产生装饰与实用 性的统一, 这样不仅仅可以符合现代人的审美, 还 可以更好的附加瑞昌竹编的审美价值。

\section{5. 结论:}

对于瑞昌竹编来说，现代产品的设计往往可以 融入到传统的民间工艺当中，将传统民间工艺当中 值得发扬和传承东西, 更好的附加到现在的产品 上, 不仅仅能够更好的去适应市场的发展, 也能够 
探索出更适合现代的产品生产模式。瑞昌竹编可以 更好的将现代产品设计当中出现的传统民间再生性 进行结合。将传统民间工艺的传承与发展，更好的 继承到民族文化传统和弘扬文化精神的宏伟目标当 中，更好地去推进瑞昌竹编的发展。

\section{项目基金}

课题：2021 年江西省文化艺术科学规划一 般项目《国家级非物质文化遗产瑞昌竹编的传 承与生产性保护研究》项目编号：YG2020121

\section{REFERENCES}

[1] Zheng Jinji, Dong Wenyuan, Zhao Minyan, Li Bei, Zheng Yan, Lu Zhenhong. Analysis on production status and market prospect of domestic bamboo tourism commodities [J]. Journal of bamboo research, 2006,25 (3): 55-58

[2] Li Yanzu. Modernity and regionality of traditional arts and crafts -- on the protection and development of traditional arts and crafts [J]. Journal of Nanjing Academy of Arts: Art and design, 2008 (1): 5-9

[3] Zhang Fuchang. Using intangible cultural heritage resources to develop characteristic tourism industry and revitalize traditional craft industry [J]. Journal of Suzhou arts and Crafts Vocational and technical college, 2008 (3): 15-20

[4] Yan Demin, Dong Wenyuan, Chen Peng, Wu wending, Xu Ming'an, Wang Minjun. Evaluation and Prospect Analysis of sustainable development conditions of bamboo industry in Suijiang County, Yunnan Province $[\mathrm{J}]$. World bamboo and rattan communication, 2009,7 (1): 1-4

[5] Chen Chong, Dong Wenyuan, Zheng Jinzhu, Duan Chunxiang, Xu Mingan, Mou Yurong. Development status and Countermeasures of follow-up industry of returning farmland to bamboo in Suijiang County, Yunnan [J]. Forestry science and technology information, 2007,39 (2): 36-38

[6] Zheng Jinji, Dong Wenyuan, Zhao Minyan, Li Bei, Zheng Yan, Lu Zhenhong. Analysis on production status and market prospect of domestic bamboo tourism commodities [J]. Journal of bamboo research, 2006,25 (3): 55-58

[7] Zhang Hao. On the design idea of folk bamboo products -- Taking the research of bamboo products in Jinyun area of Zhejiang Province as an example [J]. Art and Design: theoretical edition, 2010,0 (8x): 239-241

[8] Li Jianhui, Wu shuanghan, Li Ming, Chen Jun, Yang Wei, Yang Hua, Yang Chunping. Preliminary study on occurrence law and pollution-free control technology of bamboo leaf borer for bamboo shoots [J]. Sichuan forestry survey design, 2020 (4): 66-71

[9] Yu Wenxian, Hua Keda, Teng Ying, Jin Yahui, Zhang Yabo, Shu Jinping. Study on biological control effect of underground pests and Metarhizium anisopliae during bamboo shoot stage [J]. Zhejiang Forestry Science and technology, 2020,40 (5): 35-40

[10] Li Jianhui, Wu shuanghan, Li Ming, Chen Jun, Yang Wei, Yang Hua, Yang Chunping. Species and distribution of diseases and insect pests of bamboo shoots on the western edge of Chengdu Plain [J]. Sichuan Forestry Science and technology, 2019,40 (3): $87-91$

[11] Sun Runhe, Liu Yuan, Li Xianjun, Hou Ruiguang, Qiao JianZheng. Effect of high temperature heat treatment on bamboo bundle color and equilibrium moisture content $[\mathrm{J}]$. Journal of Central South University of forestry science and technology, 2012,32 (9): 138-141

[12] Yan Demin, Dong Wenyuan, Chen Peng, Wu wending, Xu Ming'an, Wang Minjun. Evaluation and Prospect Analysis of sustainable development conditions of bamboo industry in Suijiang County, Yunnan Province [J]. World bamboo and rattan communication, 2009,7 (1): 1-4

[13] Chen Chong, Dong Wenyuan, Zheng Jinzhu, Duan Chunxiang, Xu Mingan, Mou Yurong. Development status and Countermeasures of follow-up industry of returning farmland to bamboo in Suijiang County, Yunnan [J]. Forestry science and technology information, 2007,39 (2): 36-38

[14] Zheng Jinji, Dong Wenyuan, Zhao Minyan, Li Bei, Zheng Yan, Lu Zhenhong. Analysis on production status and market prospect of domestic bamboo tourism commodities [J]. Journal of bamboo research, 2006,25 (3): 55-58

[15] Chen Suijun. Performance analysis of bamboo processing industry development on increasing farmers' income -- Taking Fujian Yong'an bamboo plywood industry as an example [J]. Green China: theoretical edition, 2005 (06m): 61-63

[16] 伔 Chunhui, Zhang Jinze. Research on the inheritance and innovative design of folk traditional creation art in Jiangxi -- Taking Jiangxi bamboo weaving as an example $[\mathrm{J}]$. Literary life: next ten days, 2018,0 (8): 131-131 